\title{
¿Son las unidades de corta estancia médica un lugar adecuado para tratar la neumonía adquirida en la comunidad?
}

\author{
J. NOVAL MENÉNDEZ, M. T. CAMPOAMOR SERRANO, E. AVANZAS \\ GONZÁLEZ, D. GALIANA MARTÍN, J. MORÍS DE LA TASSA
}

Servicio de Medicina Interna. Hospital de Cabueñes. Gijón. Departamento de Medicina. Universidad de Oviedo. Asturias

ARE SHORT STAY MEDICAL UNITS AN APPROPRIATE PLACE TO MANAGE COMMUNITY ACQUIRED PNEUMONIA?

\section{RESUMEN}

Introducción: Evaluar los pacientes ingresados por neumonía adquirida en la comunidad (NAC) en una unidad de corta estancia médica (UCEM) para determinar, mediante los criterios de Fine para, si la reducción de la estancia en los grupos de máximo riesgo (grupos IV y V) lleva asociada una disminución de la calidad asistencial en forma de mortalidad y reingresos.

Pacientes y métodos: Se incluyeron todos los pacientes con NAC ingresados en la UCEM en un periodo de 18 meses. Se realizó un estudio univariante contrastando cada variable con la variable resultado (estancia $>0<5$ días). También se realizó un análisis multivariante en forma de regresión logística incluyendo las variables que en el estudio univariante presentaron asociación estadística.

Resultados: Se estudiaron 182 pacientes con una edad media de 73 años. La estancia media global fue de 4,3 días. El 91,2\% de los episodios se incluyeron en los grupos III $(12,1 \%)$, IV $(60,4 \%)$ y V $(18,7 \%)$ de Fine, con una estancia media para cada categoría de 4,3, 4,1 y 5,3 días respectivamente. La mortalidad global fue del $2,7 \%$ y sólo 5 pacientes reingresaron antes de 1 mes del alta. De las variables analizadas la inclusión en la categoría $\mathrm{V}$ de Fine, la presencia de hepatopatía crónica y/ó insuficiencia renal como comorbilidades asociadas, una frecuencia del pulso superior a 125 latidos por minuto al ingreso y una glucemia $>250 \mathrm{mg} / \mathrm{l}$, se relacionaron con una estancia mayor de 5 días y de ellas únicamente las 3 últimas permanecieron en el modelo final en el análisis multivariante.

Conclusiones: La NAC se puede tratar de forma eficiente en una UCEM incluso en pacientes incluidos en los grupos IV y V de Fine, apoyados en una consulta externa ágil y precoz que permita una revisión temprana.

PALABRAS CLAVE: Unidad de corta estancia médica. Neumonía adquirida en la comunidad. Criterios de Fine.
ABSTRACT

Introduction: Evaluate patients with community acquired pneumonia (CAP) admitted to our Short Stay Medical Unit (SSMU) in order to establish, using Fines's criteria, whether reducing the length of stay of maximum risk groups (IV and V) is associated with reduction of quality with either an increase of mortality or readmissions. A further objective was to pinpoint the variables associated with a prolongation of hospital stay.

Patients and methods: All CAP patients admitted to our unit over a eighteen-month period were included in the study. We conducted an univariate analysis and a step wise multivariate analysis of all the variables in the univariate analysis showing a significant statistical relation.

Results: 182 patients with a mean age of 73 years were studied. The length of hospital stay was 4.3 days. Stratified by Fines's criteria, 91.2\% were included in the groups of maximum risk: $12.1 \%$ in group III, $60.4 \%$ $I V$ and $18.7 \%$ in $V$. The medium length of stay for each category was 4.3, 4.1 y 5.3 days respectively. The mortality rate was $2.7 \%$ and only 5 patients required readmission within one month alter the discharge. Of the variables analyzed only Fine's group $V$, chronic liver disease and/or renal insufficiency as comorbidities, a pulse rate over 125 per minute and blood glucose level $>250 \mathrm{mg} / \mathrm{l}$ showed a significant relation with more than five days hospitalization, and out of them, only the three latter stayed in the multivariate analysis.

Conclusions: All CAP patients, including those in Fine's groups IV and $V$, can be treated safely and efficiently in a SSMU, providing there is an outpatient clinic for inmmediate consultation available.

KEY WORDS: Short stay medical units. Community-acquired pneumonia. Fine's criteria.

Noval Menéndez J, Campoamor Serrano MT, Avanzas González E, Galiana Martín D, Morís de la Tassa J. ¿Son las unidades de corta estancia médica un lugar adecuado para tratar la neumonía adquirida en la comunidad? An Med Interna (Madrid) 2006; 23: 416-419.

\section{INTRODUCCIÓN}

La neumonía adquirida en la comunidad (NAC) es una entidad con una elevada incidencia, se estima entre 5-10 casos/100.000 habitantes y año en el adulto (1), que se acompaña de una alta morbimortalidad. Las complicaciones y el riesgo de muerte, junto a problemas médicos, psicológicos y sociales, determinan la indicación de tratamiento domiciliario u hospitalario. Existe una gran variabilidad en los criterios de hospitalización de la NAC en relación a la población estudiada y la impresión subjetiva del clínico (1).

Fine y cols. en 1997 (2) propusieron una estratificación de

Trabajo aceptado: 20 de abril de 2006 
los pacientes mediante la Pneumonia Severity Index (PSI) con el fin de estimar el riesgo de mortalidad a los 30 días. Se ha demostrado que este índice es un excelente predictor de mortalidad, casi nula en los grupos de bajo riesgo (I a III) $(3,4)$ y que tiene un indudable valor para el clínico a la hora de decidir los ingresos hospitalarios (5). Esta y otras guías (6-8) no proporcionan información clara sobre los días de hospitalización necesarios. Existen factores ajenos a la clínica que son responsables de la prolongación de la estancia; su orientación o solución permite, de forma conjunta con un alta planificada seguida de una revisión precoz en las consultas externas, mejorar la eficiencia en la atención hospitalaria. En este sentido, la creación de unidades de corta estancia médica (UCEM) en los hospitales españoles (9-11) ha mejorado la calidad y la eficiencia en la atención de pacientes que no requieran una estancia hospitalaria prolongada.

El objetivo de nuestro trabajo es realizar un estudio retrospectivo de los pacientes ingresados por NAC en nuestra UCEM en sus primeros 18 meses de funcionamiento, para investigar si esta forma de atender a los pacientes ingresados por esta infección y estratificados mediante los criterios de Fine, se acompañó de ingresos innecesarios, y si la reducción de la estancia en los grupos de riesgo elevado (grupos IV y V) implicaba o no una disminución de la calidad asistencial con incremento de la mortalidad y de los reingresos.

\section{MATERIAL Y MÉTODOS}

Se realizó un estudio retrospectivo que abarcó un periodo de 18 meses de duración entre el 1 de junio de 2003 y el 30 de noviembre de 2004, en un hospital de área con 450 camas que atiende a una población cercana a los 300.000 habitantes. Se incluyó a los pacientes diagnosticados de NAC e ingresados en la UCEM, unidad dependiente del servicio de Medicina Interna, que dispone de 17 camas en la que la estancia máxima prevista es de 5 días. La neumonía se diagnosticó por los criterios clínicos aceptados: síntomas respiratorios e infiltrados pulmonares que no existían previamente en ausencia de otros diagnósticos.

Se revisaron las historias clínicas de los casos diagnosticados de NAC ingresados en la UCEM, cumplimentando un protocolo con las siguientes variables: edad, sexo, días de estancia, destino al alta, enfermedades asociadas (neoplasias, hepáticas, respiratorias, cardiopatía, cerebrovascular, renal crónica), exploración física (presión arterial sistólica $<90$ mmHg, temperatura $<35^{\circ} \mathrm{C} \mathrm{o}>40{ }^{\circ} \mathrm{C}$, frecuencia del pulso $>$ $125 /$ min, alteración del estado mental, frecuencia respiratoria $>30 / \mathrm{min}$ ), datos de laboratorio ( $\mathrm{pH}$ arterial $<7,35$, urea $>30$ $\mathrm{mg} / \mathrm{dl}, \mathrm{Na}<130 \mathrm{meq} / \mathrm{l}$, glucosa $>250 \mathrm{mg} / \mathrm{dl}$, hematocrito $<$ $30 \%, \mathrm{pO}_{2}<60 \mathrm{mmHg}$ ) y presencia de derrame pleural. Se consideraron excepciones a los criterios de Fine: el alcoholismo, las enfermedades neuromusculares, y el deterioro cognitivo.

Los datos fueron procesados en una base de datos Access de Microsoft.

El estudio estadístico se realizó mediante el programa SPSS/PC+ v12 (Statistical Package For Social Sciences) con licencia oficial.

Tras realizar un estudio descriptivo de las variables, se realizó un estudio univariante, contrastando cada variable con la variable resultado (estancia mayor o menor de 5 días). Por último, se realizó un análisis multivariante en forma de regresión logística, incluyendo en el modelo inicial las variables que en el análisis univariante presentaron asociación estadística relevante $(\mathrm{p}<0,05)$. El análisis se realizó mediante el sistema de pasos atrás (backward LR). Las distintas variables se codificaron respecto a la categoría de referencia. Se manejaron como probabilidad de entrada (PIN) 0,05 y como probabilidad de salida (POUT) 0,10. La clasificación final se realizó para un corte de 0,50 .

En todo el estudio se mantuvo la significación estadística para un valor de la $\mathrm{p} \leq 0,05)$.

\section{RESULTADOS}

\section{ANÁLISIS DESCRIPTIVO}

Durante los 18 meses del estudio, del total de 1.533 pacientes ingresados en la UCEM, $182(11,9 \%)$ fue con motivo de una NAC. En este periodo la estancia media global de la unidad fue de 3,9 días, y la tasa de reingresos, considerando como tales los que se producen en el primer mes tras el alta, del $6,7 \%$.

Estos pacientes diagnosticados de NAC -103 varones y 79 mujeres, con una edad media de 73 años y mediana 77 añostuvieron una estancia media de 4,3 días; en un $84 \%$ fue de 5 días o menos. La mayoría de los casos pertenecían al grupo IV de Fine $(60,4 \%)$. En la tabla I puede leerse detalladamente el porcentaje de pacientes en cada grupo y la estancia media de cada uno de ellos. Los ingresos de los dos pacientes del grupo I y de ocho del grupo II se consideraron adecuados, ya que presentaron comorbilidades que se consideraron excepciones a los criterios de Fine: ocho con insuficiencia respiratoria, uno con enfermedad neuromuscular, y deterioro cognitivo en otro paciente.

TABLA I

ESTANCIA MEDIA Y GRUPOS DE FINE

\begin{tabular}{lc}
\hline & Estancia media (días) e IC 95\% \\
\hline Grupo I & $3(15,7)$ \\
Grupo II & $3,5(2,7-4,4)$ \\
Grupo III & $4,3(3,1-5,6)$ \\
Grupo IV & $4,1(3,8-4,5)$ \\
Grupo V & $5,3(4,1-6,5)$ \\
\hline
\end{tabular}

Ciento cuarenta y tres enfermos $(79 \%)$ presentaron enfermedades asociadas; las más frecuentes fueron: cardiopatía 71 $(39 \%)$, EPOC $56(30,8 \%)$, enfermedad cerebrovascular 54 $(29,7 \%)$, diabetes $32(17,6 \%)$, neoplasia $23(12,6 \%)$, insuficiencia renal crónica $14(7,7 \%)$. Ochenta y tres pacientes $(45,6 \%)$ presentaron insuficiencia respiratoria en el momento del ingreso.

Fallecieron 5 pacientes $(2,7 \%)$, y otros 5 reingresaron en el primer mes tras el alta, dos de ellos en la primera semana. Se revisaron en la consulta externa 106 enfermos $(58,2 \%)$. 


\section{ANÁLISIS UNIVARIANTE}

Se enfrentaron las diferentes variables con la variable estancia media $>5$ días mediante tablas de contingencia de 2 por 2. De todas ellas, la relación fue estadísticamente significativa (Tabla II), en el pulso $>125 / \mathrm{min}$, la glucosa $>250$ $\mathrm{mgr} / \mathrm{dl}$, la hepatopatía crónica y la enfermedad renal crónica.

Las estancias medias para los distintos grupos de Fine fueron 3 días para Fine I; 3,5 para Fine II; 4,3 para Fine III; 4,1 para Fine IV y 5,3 para Fine V. Analizados uno a uno, el Fine $\mathrm{V}$ fue el único que se relacionó estadísticamente con la estancia mayor de 5 días (Tabla III).

TABLA II

RIESGO DE ESTANCIA MAYOR DE 5 DÍAS DE LAS DISTINTAS VARIABLES ANALIZADAS

\begin{tabular}{lcc}
\hline Variable & OR (IC 95\%) & Valor de $p$ \\
\hline Edad mayor de 75 años & $0,56(0,2-1,2)$ & 0,16 \\
Alteración estado mental & $0,68(0,1-3,1)$ & 1 \\
Presion arterial sistólica $<90 \mathrm{mmHg}$ & $1,33(0,1-12,3)$ & 0,58 \\
$\mathrm{~T}^{\mathrm{a}}<35^{\circ} \mathrm{C} 0>40^{\circ} \mathrm{C}$ & $2,75(0,4-15,8)$ & 0,24 \\
$\mathrm{pH}$ arterial $<7,35$ & $11,25(0,9-128,5)$ & 0,06 \\
Nitrógeno ureico $>30 \mathrm{mg} / \mathrm{dl}$ & $1,51(0,6-3,6)$ & 0,35 \\
Pulso $>125 / \mathrm{min}$ & $5,76(1,1-30,1)$ & 0,05 \\
Glucemia $>250 \mathrm{mg} / \mathrm{dl}$ & $5,96(1,3-25,3)$ & 0,02 \\
PaO arterial $<60 \mathrm{mmHg}$ & $0,96(0,4-2,1)$ & 0,92 \\
Hematocrito<30\% & $1,33(0,1-12,3)$ & 0,58 \\
Derrame pleural & $3,41(0,7-15,1)$ & 0,11 \\
Institucionalización & $1,05(0,1-9,3)$ & 1 \\
Neoplasia & $1,56(0,5-4,6)$ & 0,41 \\
Hepatopatía crónica & $5,76(1,1-30,1)$ & 0,05 \\
EPOC & $1,46(0,6-3,3)$ & 0,36 \\
Cardiopatía & $1,57(0,7-3,4)$ & 0,26 \\
Enfermadad cerebrovascular & $1,30(0,5-3,0)$ & 0,53 \\
Enfermedad renal crónica & $3,33(1,0-10,7)$ & 0,05 \\
\hline
\end{tabular}

TABLA III

RIESGO DE ESTANCIA MAYOR DE 5 DÍAS PARA LOS DISTINTOS ESTADIOS DE FINE

\begin{tabular}{lcc}
\hline & OR (IC 95\%) & Valor de $p$ \\
\hline Fine I & - & 1 \\
Fine II & $0,41(0,1-3,5)$ & 0,30 \\
Fine III & $0,81(0,2-2,9)$ & 0,52 \\
Fine IV & $0,65(0,2-1,4)$ & 0,30 \\
Fine V & $2,82(1,1-6,8)$ & 0,03 \\
\hline
\end{tabular}

\section{ANÁLISIS MULTIVARIANTE}

Tras introducir en el modelo inicial las variables independientes que mostraron significación estadística en el estudio univariante (pulso > 125/min, hepatopatía crónica y enfermedad renal crónica) y otras con contrastada importancia clínica (derrame pleural, edad > 75 años y EPOC), realizamos una regresión logística por pasos atrás. Las variables que permanecieron en el modelo final fueron la alteración del pulso, la glucosa $>250 \mathrm{mg} / \mathrm{dl}$ y la insuficiencia renal crónica (Tabla IV), que multiplicaban por 7 , por 6 y por 3 respectivamente el riesgo de permanecer ingresados más de 5 días.

\section{TABLA IV}

ANÁLISIS MULTIVARIANTE: MODELO FINAL TRAS REALIZAR REGRESIÓN LOGÍSTICA POR PASOS ATRÁS DESDE UN MODELO INICIAL CON LAS VARIABLES ESTADÍSTICAMENTE SIGNIFICATIVAS EN EL ANÁLISIS UNIVARIANTE

\begin{tabular}{ll}
\hline & OR $(I C$ 95\%) \\
\hline Pulso $>125 / \mathrm{min}$ & $7,7(1,4-41,2)$ \\
Glucosa $>250 \mathrm{mg} / \mathrm{dl}$ & $6,6(1,5-29,4)$ \\
Insuficiencia renal crónica & $3,7(1,1-12,6)$ \\
\hline
\end{tabular}

\section{DISCUSIÓN}

Nuestro centro como todos los hospitales públicos de España, ha sufrido en los últimos años un cambio en la población que atiende, que se caracteriza por un incremento en la edad media y la concurrencia en el mismo paciente de varios procesos patológicos crónicos, que condicionan una necesidad mayor de recursos. Ello ha favorecido la búsqueda de alternativas a la hospitalización convencional en busca de una mayor eficiencia.

La creación y desarrollo de una UCEM como unidad diferenciada dentro del servicio de Medicina Interna nos ha permitido obtener una reducción de la estancia media con una baja tasa de reingresos.

Tras analizar los criterios de ingresos de los pacientes afectos de NAC en nuestra serie, sólo cinco de ellos se consideraron como innecesarios; la tasa de reingresos $(2,7 \%)$ ha sido más baja que las descritas $(3,4 \%)$ (12).

La duración del tratamiento antibiótico en la NAC debe de ser individualizado y dependiente del tiempo en el que el paciente se estabiliza clínicamente. Si consideramos a la temperatura como un buen indicador de mejoría clínica o estabilidad, los tratamientos cortos de 5 ó 7 días de duración pueden ser apropiados para la mayoría de los pacientes. Para esta entidad, se ha estimado el tiempo de defervescencia en 3 días (13). Estudios in vitro han demostrado que una elección antibiótica adecuada reduce significativamente el número de microorganismos en las primeras 24 horas de tratamiento (14). Dunbar et al, en su reciente trabajo mostraron una respuesta clínica y bacteriológica similar en los pacientes tratados 5 y 10 días (15). Una terapia secuencial con paso rápido de tratamiento antibiótico de la vía intravenosa a la vía oral influye significativamente en la reducción de la estancia hospitalaria. La excelente absorción oral de los antibióticos actualmente disponibles lo permite, en cuanto se produzca la estabilidad clínica y con ello el alta hospitalaria precoz.

Otro factor que con frecuencia prolonga los días de estancia en la NAC es el control radiológico. La imagen radiológica no es un buen indicador de la evolución ya que, en los pri- 
meros días, puede no modificarse o empeorar incluso ante una mejoría clínica evidente. La única indicación de repetirla precozmente es ante la sospecha de complicación o un empeoramiento clínico. De hecho sólo en el $51 \%$ de los pacientes se resuelve en las dos 2 primeras semanas tras el inicio del tratamiento (16).

Como conclusión, a la luz de los datos expuestos, consideramos que la NAC puede ser tratada de forma eficiente en una

\section{Bibliografía}

1. Mandell LA. Community-acquired pneumonia. Etiology, epidemiology and treatment. Chest 1995; 108: 35S-42S.

2. Fine MJ, Auble TE, Yealy DM, Hanusa BH, Weissfeld LA, Singer DE, et al. A prediction rule to identify low-risk patients with communityacquired pneumonia. N Engl J Med 1997; 336: 243-250.

3. Querol-Ribelles JM, Tenias JM, Querol-Borrás JM, González-Granda D, Hernández M, Ferreruela R, et al. Validación del Pneumonia Severity Index en la hospitalización de los pacientes con neumonía adquirida en la comunidad. Med Clin (Barc) 2004; 122: 481-486.

4. España PP, Capelastegui A, Quintana JM, Soto A, Gorordo I, GarcíaUrbaneja M, et al. A prediction rule to identify allocation of impatient care in community-acquired pneumonia. Eur Resp J 2003; 21: 695701.

5. Ruiz A, Vallverdú M, Falguera M, Pérez J, Cabré X, Almirall M, et al. Neumonía adquirida en la comunidad: impacto de la utilización de una estrategia terapéutica basada en la mortalidad a corto plazo. Med Clin (Barc) 1999; 113: 85-88.

6. American Thoracic Society. Guidelines for the initial management of adults with community-acquired pneumonia: diagnosis, assessment of severity, and initial antimicrobial therapy. Am Rev Respir Dis 1993; 148: 1418-1426.

7. Bartlett JG, Breinman RF, Mandell LA, Fine TM. Community-acquired pneumonia in adults: guidelines for management. Clin Infect Dis 1998; 26: 811-838.

8. Dorca J, Bello S, Blanquer J, de Celis R, Molinos L, Torres A, et al. Diagnóstico y tratamiento de la neumonía adquirida en la comunidad. Arch Bronconeumol 1997; 33: 240-246.
UCEM, incluso en las categorías de mayor gravedad (grados IV y V de Fine).

\section{AGRADECIMIENTOS}

Al Dr. Gonzalo Solís del Servicio de Pediatría de nuestro centro, por su inestimable ayuda en el estudio estadístico del trabajo.

9. Marco J, Jusdado JJ, Plaza S, Rondon P, Torres R, Solís J. Diseño e implantación de una Unidad Médica de Hospitalización de corta estancia. Repercusión y actividad asistencial. Gestión y evaluación de costes sanitarios 2002; 3: 64-73.

10. Barbado MJ, Jimeno A, Estolaza JM, Molinero J. Unidad de corta estancia médica dependiente de Medicina Interna. An Med Interna (Madrid) 1999; 16: 504-510.

11. De la Iglesia F, Pelliter C, Ramos B, Castro B, Rodríguez A, Diz-Lois F. La unidad de corta estancia médica (UCEM) de La Coruña: nuestra experiencia. An Med Interna (Madrid) 1997; 14: 125-127.

12. Menéndez R, Ferrando D, Valles JM, Martínez E, Perpiña M. Initial risk class and length of hospital stay in community-acquired pneumonia. Eur Resp J 2001; 18: 151-156.

13. Halm EA, Fine MJ, Marrie TJ, Coley CM, Kapoor WN, Obrosky DS, et al. Time to clinical stability in patients hospitalized with communityacquired pneumonia: implications for practice guidelines. JAMA 1998; 279: 1452-1457.

14. Lacy MK, Lu W, Xu X, Tessier PR, Nicolau DP, Quintiliani R, et al. Pharmacodynamic comparisons of levofloxacin, ciprofloxacin, and ampicillin against Streptococcus pneumoniae in an in vitro model of infection. Antimicrob Agents Chemother 1999; 43:672-7.

15. Dunbar LM, Wunderink RG, Habib MP, Smith LG, Tennenberg AM, Khashab MM, et al. High-dose, short-course levofloxacin for community-acquired pneumonia: a new treatment paradigm. Clin Infect Dis 2003; 37: 752-60.

16. Mittl RL Jr, Schwab RJ, Duchin JS, Goin JE, Albeida SM, Miller WT. Radiographic resolution of community-acquired pneumonia. Am J Respir Crit Care Med 1994; 149: 630-635. 